Historic, Archive Document

Do not assume content reflects current scientific knowledge, policies, or practices. 


\section{Catalog of}

Fine Peonies and

Oriental Poppies

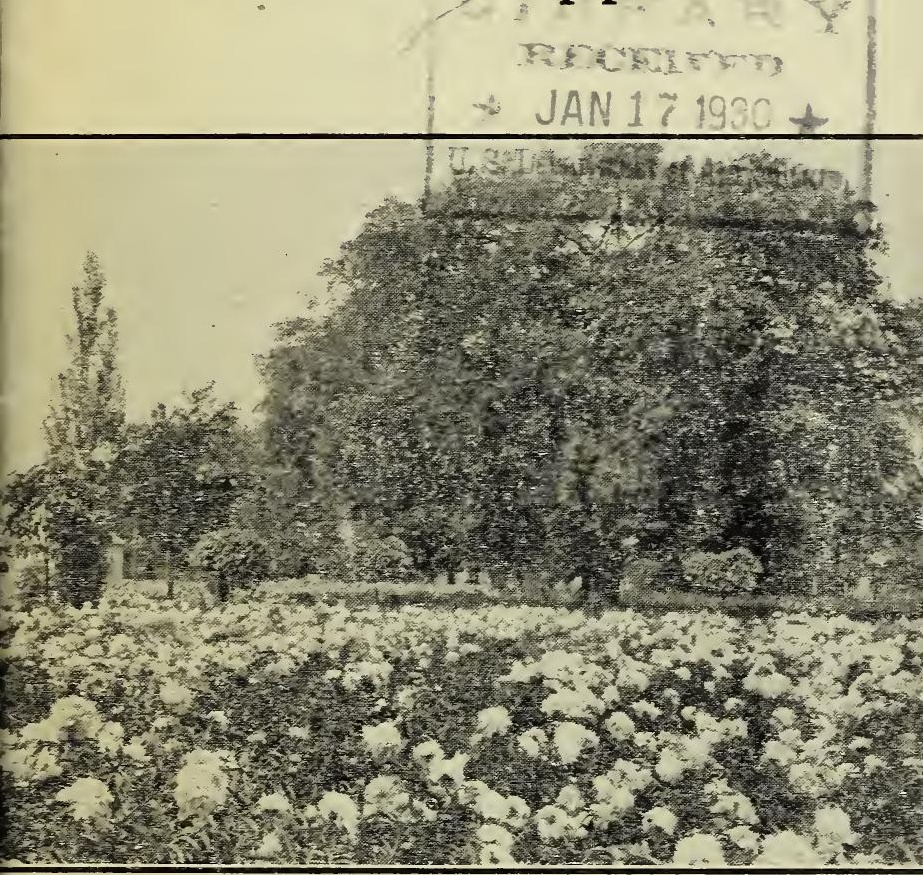

Wahnfried Gardens

L. J. Germann

Van Wert, Ohio 


\section{FOREWORD}

Wahnfried Gardens have passed their twentyeighth birthday, which makes them the oldest Peona Gardens in Van Wert. From a modest beginning, our collection has been added to each year, and at this time it includes nearly all of the world's best. We are located on South Washington and Elm Streets, in the seventh block due South from the Public Square. Visitors are always welcome.

\section{Peonies}

\section{ARRANGEMENT AND VARIETIES}

We have arranged the varieties in alphabetical order. The figures at the left indicate their rating as established by The American Peony Society on a basis of 10 as perfect. Where no figures appear no rating has been given. The name of the variety is followed by the name of the Originator, and this by the color of the bloom. The rating should be of great assistance in making selections.

\section{PRICES}

The prices quoted are for roots from three to five eyes, and guaranteed to be true to name. Each root has my personal attention. If an error should occur the same is cheerfully rectified. We do not substitute unless instructed to do so.

\section{SHIPPING TIME}

We begin to dig Peonies as early in September as possible and continue until the ground freezes. The Fall is the best time to plant.

\section{TERMS}

Cash with order. Small orders we send by Parcel Post and large ones by Express. Write plainly your Express Office and any other shipping instructions.

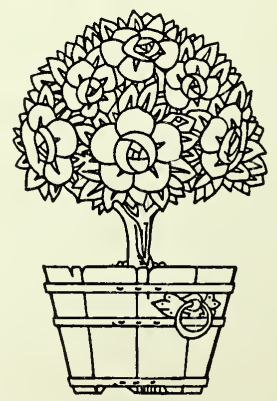




\section{Peonies}

Div. 3 to 5 Eyes

7.f Adaice Hollis (Hollis) Blush white.....\$ 2.00

s. Iddielanclea (Brand) Creamy white..... 8.00

i.t Idmiral Togo (Hollis) Iark crimson..... 1.00

(.) Irolph liosseau (I essert) Deep garnet... 1.50

Alisaben (Rosefield) Bright red........ 1.00

S.7 Alhatre (Crousse) Shell pink................... 1.00

8.6 Ilbert Crousse (Crousse) Shell pink...... 1.00

8.8 Alsace Lorraine (Lemoine) Creamy white 2.50

Alice Harding (Lemoine)...............10000

8.6 A. P. Sauniers (Thurlow) Flesh pink.... 10.00

7.. Aic.ie Brand (Brand) Shell pink........ 1.50

S.1 Arlequin (Dessert) Lilac pink and straw

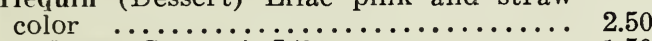

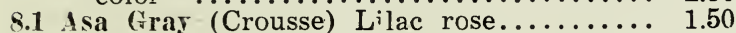

8.7 ugust Dessert (Lessert) Deep rose..... 7.50

8.1 Aurore (Dessert) Lilac white........... 1.00

8.6 Aralanche (Crousse) Ivory white........... 1.00

8.0 Ariateur Raymond (Dessert) Cherry red.. 2.00

88 Ball 0'Cotton (Franklin) Pure white..... 10.00

9.0 Baroness Schroeder (Kelway) Lovely white 1.50

8.7 Bayedere (Lemoine) Cream white....... 4.00

Bay State (Hollis) Attractive red........ 1.50

7.9 Beauty's Mask (Hollis) Lilac pink........ 1.50

8.6 Bertrade (Lemoine) Ivory white........ 3.00

Belroit, Dark red ..................... 3.00

8.7 Brand's Magnificent (Brand) Deep dark red $\ldots \ldots \ldots \ldots \ldots \ldots \ldots \ldots \ldots \ldots \ldots \ldots$.

8.4 Candeur (Dessert) Rose pink and white..

7.S Chas. Mckellip (Brand) Rich crimson.... 2.50

8.5 Chas. Neidel (Wett) Blush pink......... 2.50

Cecelia Kelway (Kelway) Mauve pink..... 2.50

8.7 Cherry Hill (Thurlow) Deep garnet..... 4.00

8.4 Chestine Gowdy (Brand) Pink and cream 1.50

8.6 Clair Dubois (Crousse) Satiny pink....... 1.00

Claude Galle (Lemoine) Cream white.... 1.00

8.5 Clemenceau (Dessert) Carmine......... 5.00

8.1 Cournne d, 'Or (Calot) Milk white......... 1.00

Crimson Victory (Rosefield) Deep red.... 1.00

8.4 David Harum (Brand) Bright red........ 6.00

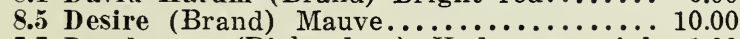

7.7 Dorchester (Richardson) Hydrangea pink 1.00

8.1 Duchess deNemours (Calot) White-green reflex ........................ 1.00

9.1 E. C. Shaw (Thurlow) Flesh pink.......... 20.00

7.6 Edulis Superba (Lemoine) Rose pink and early ........................... 1.00

7.5 Edwin Forrest (Hollis) Dark crimson.... 2.00

8.7 E. J. Shaylor (Shaylor) Rose pink....... 15.00

9.1 E. B. Browning (Brand) Ivory white..... 4.50

8.7 Elwood Pleas Pleas) Lovely clear pink. . 2.50

9.0 Enchantment (Hollis) Deep shell pink... 1.50

8.1 Enchantress (Lemoine) Creamy white.... 4.00

Enfant de Nancy (Crousse) Pale lilac rose.

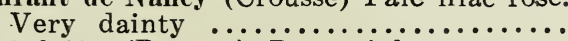

7.9 .

2.00

8.0 Etta (Terry) Shell pink.............. 1.50

8.3 Eugene Verdier (Calot) Deep shell pink... 2.00

8.6 Eugenie Verdier (Calot) Hydrangea pink 1.00

8.3 Evening Glow (Hollis) Lilac............. 3.00

Excelsior (Terry) Large deep red blooms 1.00

8.5 Exquisite (Kelway) Rose pink.......... 3.00

7.5 Fanuy Crosby (Brand) Delicate pink and

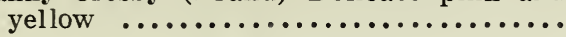


Div. 3 to 5 Eyes

7.8 Faribault (Brand) Deep rose and silver.. 2.00

8.4 Felix Crousse (Crousse) Brilliant red..... 1.00

9.3 Festiva Iraxima (Meillez) Large white blooms ....................... 1.00

8.1 Florence Nightingale (Brand) White.... 1.00 Floweret of Eden (Dr. Neeley) ......... 20.00

8.7 Frances Shaylor (Shaylor) White-yellow stamens ..................... 400

9.1 Frances Willard (Brand) Blush white... 3.00 Fraglans, Rose pir $\mathrm{k}$ and very fragrant... 1.00

8.1 Galathee (Lemoine) Pearly white....... 3.50

8.9 Georgianna Sl'avlor (Shaylor) Rose pink.. 3.00

8.2 figantea (Calot) Lilac rose............. 1.00

8.6 Ginette (Dessert) Deep flesh-salmon tints 2.00

8.2 Gismonda (Crousse) Flesh color.......... 1.00 Golten Dawn (Gumm) Straw color, ruby

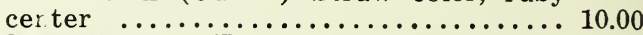

Golden Nuggett (Pleas) Rosy lilac, yellow collar ......................... 3.00

Golden Wedding (Pleas) Canary........... 2.00

9.2 Grace Loomis (Saunders) Clear white... 25.00

8.1 Grandiflora NiveaPlena (Richar son) Cream

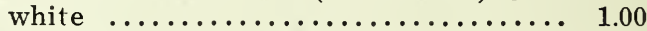

8.8 Grandiflora (Richerdco.s) B icht shell p nk 1.50

8.2 Grover Cleveland (Terry) Crimson, very large blooms .................... 1.50

Hansina Brand (Brand) Flesh white.... 50.00

8.2 H. A. Hagen (Richardson) Violet rose.... 2.75

Harriet Becclser Stowe (Hollis) Hydrangea pink which fades to white........ 3.00

Harriet Beecher Stowe (Hollis-Anderson) Cream white with golden stamens.... 15.00

H. R. H. Princess Louise (Kelway) Shell pink .......................... 1.50

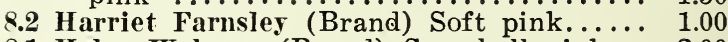

8.1 Helen Wolaver (Brand) Sea shell pink... 2.00

8.8 Henry Avery (Brand) Pink and creamy

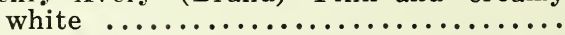

Henri Merger (Crousse) Pure mauve..... 1.00

8.1 Hercules (Terry) Pink with stamens..... 1.00

7.8 H. F. Reddick (Brand) Dark crimson..... 1.00

Irma (Calot) Violet rose.............. 1.50

Ivanhoe (Pleas) Tall dark red........... 2.00

8.9 James Boyd (Thurlow) Flesh pink....... 7.50

8.7 James Kelway (Kelway) Large white blooms ....................... 1.50

8.7 James R. Mann (Thurlow) Deep ivory pink 1500 Jeanne Gaudichau (Mil) White......... 2.00

Jennie E. Richardson (Hollis) Pink and

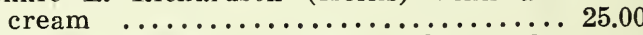

9.2 Jeannot (Dessert) Flesh pink-lavender

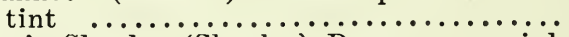

8.6 Jessie Shaylor (Shaylor) Deep cream-pink tinge.$\ldots \ldots \ldots \ldots \ldots \ldots \ldots \ldots \ldots \ldots \ldots$.

8.2 John Richardson (Richardson) Blush rose

8.9 Jubilee (Pleas) Flesh white-opalescent

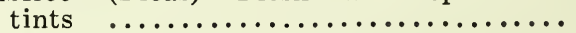

8.6 Judge Berry (Brand) Soft light pink.....

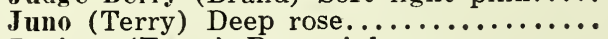

Jupiter (Terry) Rose pink.............

8.8 Karl Rosefield (Rosefield) Lovely red.....

9.0 Katherine Havemyer (Thurlow) Large

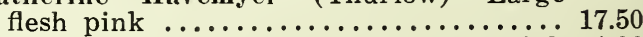

Kelway's False Queen (Kelway) Rose pink 1.00

9.8 Kelway's Glorious (Kelway) Large cream white $\ldots \ldots \ldots \ldots \ldots \ldots \ldots \ldots \ldots . \ldots . \ldots 12.00$ 
Div. 3 to 5 Eyes

Koenigswinter ( $\mathrm{G} \& \mathrm{~K}$ ) Large pink..... 4.00

9.1 Lady Mlexandra Ii ufi (helway) Rose to ivory white .................. 2.00

Lady Nlexandra Dufí No. 2 (Kelway) Large shell pink........................ 1.50

7.S Lady Beresforl (Kelway) Blush pink.... 1.50

7.9 Lady Garrington (Kelway) Silvery rose

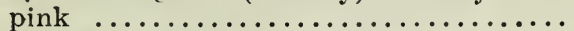

8.6 Lady Emily (Pleas) Ivory rose pink......

Lady Romily (Kelway) Sea shell pink....

7.8 Lafayette (Dessert) Salmon pink.......

9.2 LaFee (Lemoine) Mauve rose............

9.0 LaFrance (Lemoine) Rose white..........

8.5 Laperle (Crousse) Lilac white.............

8.3 LaRoserie (Crousse) Pure white gold sta-

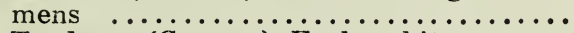

8.1 LaTendress (Crousse) Early white........ LaVerne (Kelway) Ieep shell pink......

8.4 Lamertine (Lemoine) Leep carmine rose Lamertine (Calot) Rcse pi.k............

8.8 Laura Dessert (Dessert) Cream white and

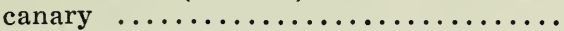

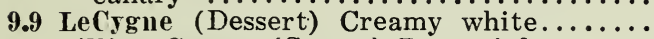
Lillian Gumm (Gumm) Rose pink........

8.1 Livingston (Calot) Light pink..........

9.0 Longfellow (Brand) Cherry red..........

8.4 Lora Dexleimer (Brand) Crimson.........

7.8 Louisa Brand (Brand) Pink to blush white

8.8 Loreliness (Hollis) Hydrangea to white...

8.1 Lucy E. Hollis (Hollis) Soft rose pink....

8.7 Luettea Pfeifier (Brand) Large light pink

9.0 Mabel Franklin (Franklin) Light pink, darker toward center.............. 10.00

8.3 Marcelle Dessert ( 1 essert) White and 1 lac 1.00

7.5 Marshal Valliant (Calot) Lilac white.... .75

8.1 Marguerite Gaudicl:au (Millet) Shell pink $\mathbf{2 . 0 0}$

8.4 Marguerite Gerard (Crousse) Pale hy-

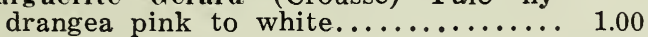

8.1 Marie (Calot) Lilac white............. 1.00

8.9 Marie Crousse (Crousse) Deep flesh color 2.00

8.3 Marie Jaquin (Verdier) Delicate cream.. 1.50

8.5 Maric Lemoine (Lemoine) Pure white.... 1.00

Marie Houillon (Calot) Large white peona 1.00

7.8 Marie Stewart (Verdier) Lavender chang-

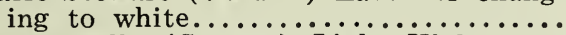

Marmontelle (Crousse) Light Violet rose

9.1 Martha Bulloch (Brand) Large blooms of

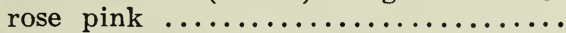

7.9 Mary A. Livermore (Hollis) Lilac pink

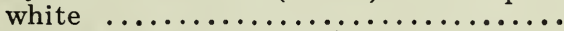

7.5 Mary Baker Eddy (Hollis) Rose pink....

8.7 Mary Brand (Brand) Bright crimson.....

9.4 Mary Woodbury Shaylor (Shaylor) Shell

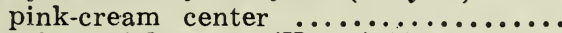

8.5 Mand L. Richardson (Hollis) Flesh pink..

7.9 Mathilde Roseneck (Crousse) Lilac rose...

7.3 Midnight (Brand) Deep marroon........

8.5 'Mid Summer Night's Dream (Pleas) Ivory

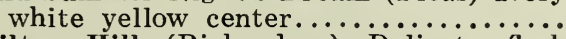

9.0 Milton Hill (Richardson) Delicate flesh

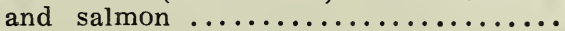

Miriam (Chase) Pink with yellow stamens

7.8 Miss Salway (Kelway) Lilac white........

7.8 Model de Perfection (Crousse) Violet rose

7.8 Modiste Guevin (Guerin) Solferino red...

8.6 Madam August Dessert (Dessert) Violet 
Div. 3 to 5 Eyes

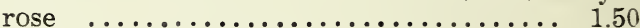

7.8 Mnie. Boulanger (Crousse) Glossy pirk... 100

7.8 Mme. Bucquet (Dessert) Dark crimson... 1.00

8.1 Ime. Calot (Niellez) Hydrangea pink.... 1.00

7.9 Mme. Camile Bancel (Crousse) Deep rose

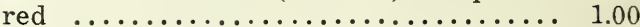

7.9 Mme. Crousse (Calot) Pure white........ 100

8.2 Ime. D. Treyeran (Dessert) Rosy white.. 200

7.9 Mme. De Vernrille (Crousse) Blush white 1.00

Mme. Ducel (Mechin) Silvery lilac pink.. 1.00

8.5 Mme. Galle (Crousse) Delicate lavender

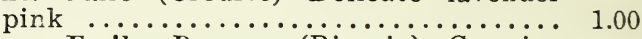

7.4 Ine. Emile Dupraz (Riverie) Carmine

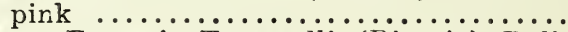

Ime. Francois Toscanelli (Riverie) Eeli-

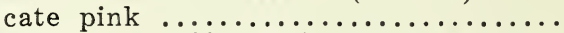

7.9 Mme. Geissler (Crousse) Rose pirk.....

9.4 Ime. Jules Dessert (Dessert) White and

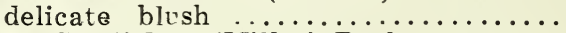

8.8 Mme. Gandichau (Millet) Dark garnet....

8.1 Mme. Leomie Calot (Calot) Shell p:nk...

7.7 Ime DeVatry (Guerin) Lilac white and

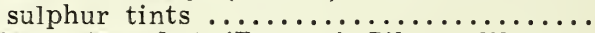

8.5 Mme. Manchet (Dessert) Silvery lilac.... Mlle. Jeamne Riverie (Riverie) Delicate

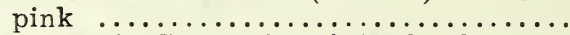

7.4 Mile. Marie Calot (Calot) Milk white...

8.3 Mons Jupont (Calot) Ivory white.......

9.2 Mons Jules Elie (Crousse) Large pink-silvery sheen..$\ldots \ldots \ldots \ldots \ldots \ldots \ldots$.

8.8 Mons Martin Cahuzac (Dessert) Darkest

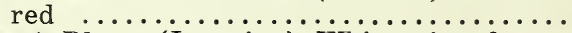

8.4 Mont Blanc (Lemoine) White tinted rose

8.2 Moses Hull (Brand) Dark shell pink.....

8.3 Mrs. A. G. Ruggles (Brand) Light pink...

Irs. 1. Brand (Brand) Pure white... 5000

7.8 Mrs. Carew (Brand) Silvery blush....... 1.00

9.2 Mrs. C. S. Minot (Mirot) Shell pink-tinted

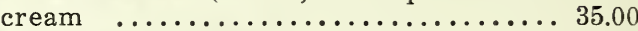

9.3 Mrs. Edward Harding (Shaylor) Lovely

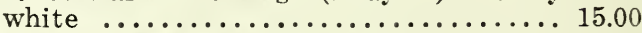

8.4 Irs. John Smith Fogg (Hollis) Light pink 200

8.2 Mrs. John M. Lewis (Lewis) Dark red.... 3.00

IIrs. George Bungard (Kelway) Shell pirk 5.00

8.8 Mrs. M. P. Clough (Shaylor) Salmon pink 350

9.0 Mrs. Shaylor Force (Shaylor) Ivory white 12.00

7.0 Multiflora (Pleas) Silvery pink......... 1.50

9.0 Nina Secor (Secor) Pure white........... 10.00

9.1 Nymphae (Thurlow) Delicate pink...... 3.00

8.5 Octavie Demay (Calot) Lovely flesh pink 1.00 Odette (Lemoine) Lilac rose splashed pink 1.00

Ofïicinalis Rubra Plena. Very early bright

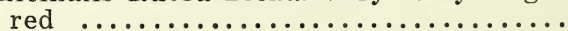

7.6 Old Silver Tip (Brand) Madder rose with

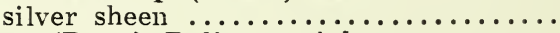

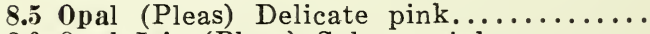

8.0 Opal Iris (Pleas) Salmon pink..........

Ornament de Massives. Cream and straw

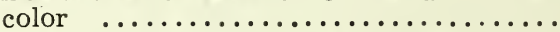

8.5 Paradise (Hollis) Hydrangea pink.......

8.1 Pasteur (Crousse) Delicate pink..........

8.0 Perfection (Richardson) Lilac white and

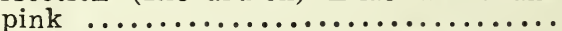

7.6 Petite Renee (Dessert) Light magenta...

8.6 Peter Pan (Hollis) Lilac rose...........

7.7 Philomele (Calot) Violet rose-yellow cen- 
Div. 3 to 5 Eyes

ter 1.00

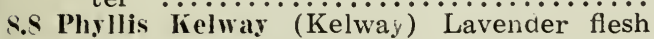
shading to white............... 9.00

9.2 Phillipe Rivoire (Rivere) Fine dark crimson $\ldots \ldots \ldots \ldots \ldots \ldots \ldots \ldots \ldots \ldots . \ldots \ldots . \ldots \ldots . \ldots \ldots$

Pink Lady (Pleas) Lainty pirk......... .50 8.S Plocbe Carey (Brand) Rose pink....... 5.00

7.6 Pierre Dessert (Lessert) Dark red....... 1.00

\$.2 Pierre Iincharte (Crousse) Lovely shell

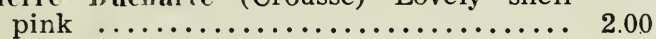

¡.6 Pierre Reinoux (Dessert) Bright rose.... 1.50

7.5 Pres. Rooserelt (Warnaar) Brilliant dark

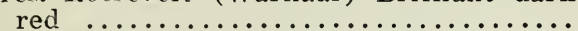

8.7 Pres. Tait Reine Hortense (Calot) Large

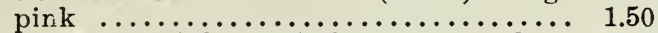

9.3 Pres. Wilson (Thurlow) Soft rose pink... 25.00

\$.9 Pride of Essex (Thurlow) Flesh pink..... 6.00

Pride of Paulding (Dr. Neeley) Pink..... 25.00

8.6 Primerere (Lemoire) Cream yellow and

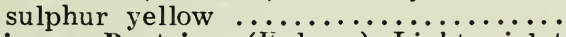

7.4 Prircess Beatrice (Kelway) Light violet

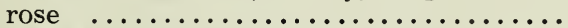

Oneell Victoria. Large white tinted pink

6.3 Queen of Pleasance (Pleas) Rose and sal-

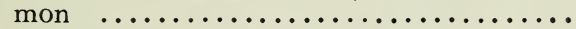

Quaker Lady (Pleas) Blush white with

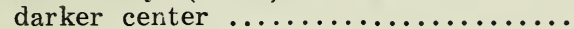

\$.3 Rachel (Lemoine) Fresh soft rose.......

¡.9 Rachel (Terry) Good red Peony..............

9.0 Raoul Dessert (Dessert) Mauve, shaded

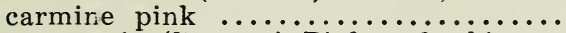

8.4 Reene Marie (Lessert) Pink and white...

7.s R. P. Whitfield (Richardson) Delicate hydrangea pink

S.8 Richard Carvel (Brand) Very läge bright

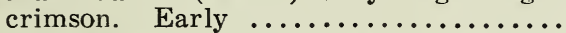

9.0 Rosa Bouheur (Dessert) Light violet rose

9.1 Rose Shaylor (Shaylor) Delicate flesh

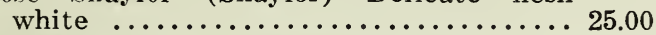

8.S Rosette (Dessert) Silvery shell pink..... 4.50

7.6 Rosy Iawn (Pleas) Bright pink, full flow-

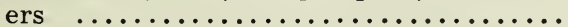

Rubra Superba (Richardson) Crimson....

8.1 Ruth Brand (Brand) Soft lavender pink

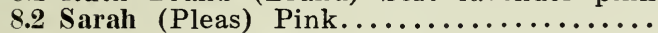

9.0 Sarah Bernliardt (Lemoine) Apple blos-

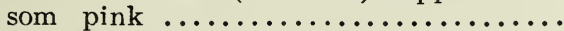

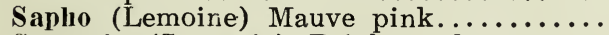

Sarcoxie (Sarcoxie) Bright red..........

8.5 Saral Carstonsel (Terry) Red..............

9.1 Sarah K. Thurlow (Thurlow) White and

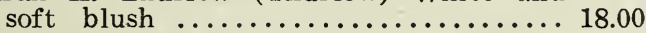

9.1 Secretary Fewkes (Shaylor) Cream white with lighter center............... 6.00

8.5 Shaylor's Iream (Shaylor) Flesh white...

Shebona (Harrison) Bright red. Very tall

7.7 Sistel's Ammie (Brand) Sea shell pink...

9.7 Solange (Lemoine) Waxy cream deepening to a golden $\tan . \ldots \ldots \ldots \ldots \ldots \ldots \ldots \ldots$

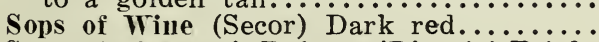

7.6 Souv. de Francois Ruitton (Riverie) Bright

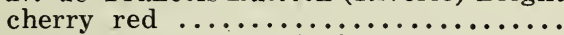

Souv. de L'Exposition Universelle (Calot)

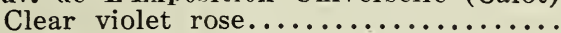

8.8 Souv. de Louis Bigot (Dessert) Bengal rose salmon pink and silver............. 
Div. 3 to 5 Eyes

8.8 Standard Bearer (Hollis) Light violet rose 5.00

7.8 Stephonie (Terry) Delicate pirk shading

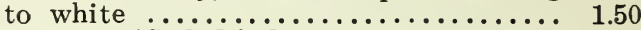

8.4 Strassburg $(G \dot{G} \mathrm{~K})$ Silvery lilac rose.... 3.00

7.3 Supreme (Hollis) Late pink........... 1.50

8.2 Suzette (Dessert) Bengal rose......... 3.00

7.7 Suzanna Dessert (Dessert) Pink with sil-

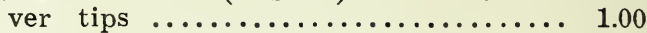

8.3 The Gem (Pleas) Tall bright red....... 2.00

9.8 Therese (Lessert) Large shell pink...... 3.50

9.1 Thomas C. Thurlow (Thurlow) Salmon

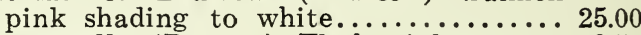

9.4 Tourangelle (Dessert) Flesh pink....... 3.50

7.8 Triumpl de Exposition d'Lille (Calot) Pale pink with darker shades....... 1.00

7.4 Umbellate Rosea (Dessert) Violet rose

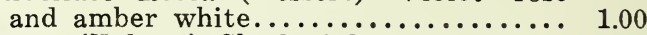

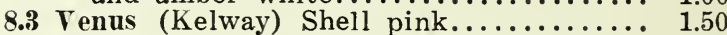

Veribest (Hollis) Milk white. Very late.. 1.50

8.3 Victor de Laniarne (Dessert) Amaranth red with silver reflex.............. 4.00

Ville de Nancy (Calot) Carmine rose... 1.00

7.6 Virginie (Calot) Light pink........... 1.00

9.3 Walter Faxou (Richardson) Lovely clear

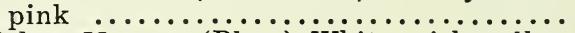

8.0 Walter Morgan (Pleas) White with yellow

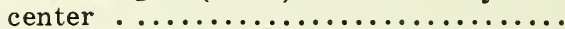

7.9 Welcome Guest (Hollis) Bright rose chan-

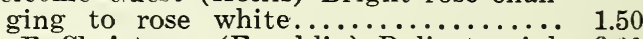

8.8 W. F. Christman (Franklin) Delicate pink 6.00

William Messman. A lovely double white 1.50

8.4 William F. Turner (Shaylor) Very dark

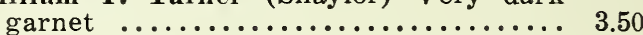

8.8 Wilton Lockwood (Shaylor) Deep pink with crimson markings.............. 10.00

7.8 Winnifred Domme (Brand) Bright uniform crimson ........................... 2.00

Zest (Hollis) Silvery rose pink. Very late 1.50

\section{Peony Collections}

We receive many letters asking our opinion and advice as to varieties of Peonies and solicit our aid in making selections. These letters are not only appreciated but invited, and any service we can render along these lines is not only a privilege but a great pleasure. For convenience and consideration we have compiled a number of collections as follows:

COLLECTION A-MRS. PLEAS VARIETIES

1 Jubilee ..................\$2.00

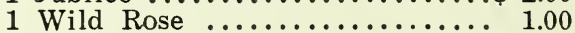

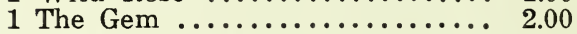

1 Queen of Pleasance ......... 2.00

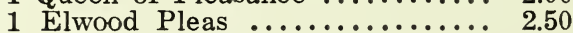

1 Opal ........................... 2.00

$\$ 11.50$ For $\$ 9.00$

\section{COLLECTION B}

1 Elwood Pleas .............. \$ 2.50

1 Venus ........................ 1.50

1 Octavie Demay ............. 1.00

1 The Gem ................. 2.00

1 Festiva Maxima ................ 1.00

1 Mada August Dessert ........ 1.50 
1 Etta

Div. 3 to 5 Eyes 1.50

\section{COLLECTION C}

1 Frances Willard ............. \$ 3.00

1 Madam Jules Dessert ......... 3.00

1 Lady Alex. Duff ............. 2.00

1 Therese .................. 3.50

1 Jubilee $\ldots \ldots \ldots \ldots \ldots \ldots \ldots \ldots \ldots, 2.00$

$\$ 13.00$ For $\$ 10.00$

COLLECTION D

1 LeCygne .................. \$ 8.00

1 Kelway's Glorious ............ 12.00

1 Georgiana Shaylor .......... 3.00

1 Walter Faxon .............. 3.50

1 Solange .................. 3.50

1 Souv. de Louis Bigot ......... 4.00

1 Therese .................. 3.50

1 Richard Carvel ............. 4.50

$\$ 42.00$ For $\$ 35.00$

\section{Japanese Peonies}

The Japanese Peonies are those whose blooms have one or two rows of petals and a cushion of yellow stamens heavier than in the Single Peonies. They are very desirable for cut flowers and are very easy of artistic arrangement.

$\$ .1$ tkalu Carmine red ................\$ 3.00

s.0 Alma (Shaylor) Delicate pink........... 3.00

S.0 Altar Candies (Pleas) Delicate pink, straw color ....................... 3.00

Ama-NoSoDo Bright rose yellow center.. 10.00

Cathedral Deep pink ............... 3.00

Estler (Terry) Light rose............... 1.00

9.2 Furajo Mahogany red ................ 6.00

HaNoNoSoto Deep pink ............... 3.50

Hart P. Danks Rose color ............... 2.50

Hettie Elliott Hydrangea pink. Stamens 2.00

Hinoke-Saki Violet rose ............. 3.00

Isani-Gidui Ivory white and gold....... 15.00

Kumagoe Carmine pink ............. 1.50

Magnificent (Kelway) Rose color......... 3.00

Moonyeen Clair (Pleas) Rose pink and gold 4.00

Mikado (Barr) Crimson and gold........ 2.50

Noon Day Violet rose. Petaloids same color edged gold .................. 5.00

Norelty (Pleas) Rose and straw color..... 2.00

ostrich Plume White with cream center 1.50

Rare Brocade Cream white with green reflex

1.00

Snow Wheel Pure white, yellow center... 5.00

9.4 Tomatbaco Light pink ............. 20.00

\$.9 Tokio Clear pink .................. 6.50

8.0 Torpilliur Carmine ................. 4.00

\section{Single Peonies}

Single Peonies are those whose blooms have one row of petals and a cushion of yellow stamens. They are very attractive and especially so in the morning and evening when the blooms almost close giving a rosebud effect. Very graceful for cut flowers. 
Single and Jap. varieties are becoming more popular each season.

Camille (Dessert) Violet purple, golden stamens ....................... 1.50

Cottage Maid (Pleas) Shell pirk ........ 1.00

Clio (Peterson) Pirk, golcen stamens.... 1.00

Harriet Olney (Brand) Soft rose color,

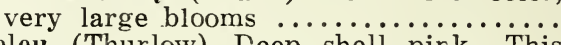

9.0 Helell (Thurlow) Deep shell pink. This variety has a double row of petals. Cen-

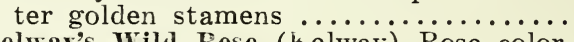

Kelway's Wild Rose (helway) Rose color, yellow stamens ....................

Knight of the Thistle (Kelway) Rich crim-

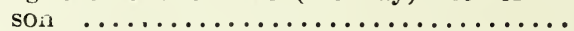

L'Etencelante (Iessert) Clear carmine...

LeJour (Shaylor) Large pure white, yellow stamens ................... 4.00

8.5 Marguerite Dessert (Dessert) White.... 4.00

Iinnehaha Dark red ................ 2.50

Lady of the West (Kelway) Large white.

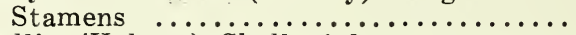

Nellie (Kelway) Shell pink ..............

Pecalontas Cherry red .................

Pleas queen Large white blooms..........

Prairie Rose (Pleas) Resembles wild rose.

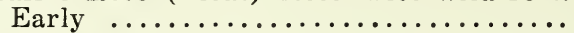

Pride of Langport (Nelway) Peach pink.

Pure Lore Firk fades to white.........

Rose Caralier (Pleas) Magenta rose......... 1.00

Spring Maid (Pleas) Brilliant pir....... 1.00

Wild Rose (Pleas) Resembles pink wild

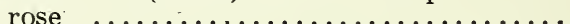

\section{Oriental Poppies}

\section{FINEST IN THE WORLD}

"Eclipsing with its gorgeousness the lily and the rose,

The Oriental poppy in my quiet garden grows.

A brazen beauty of the East from some zenana strayed,

In scarlet silk and sable fringe most stunningly arrayed."

Oriertal Poppies are the regal representatives of this popular genus growing 3 to 4 feet high, and far surpassing in splendor of bloom all the annual and biennial kinds. For gorgeous display of rich and brilliant coloring nothing equals them during their flowering season in May and June. They can be planted singly or in masses, and their large flowers and freedom of bloom render them conspicuous in any position. They begin to bloom after the passing of the tulips and other early Spring bulbs, and make a royal leader for that triple successionthe Iris, the Peona and the Rose. They are easy of culture and every garden should possess a collection of these beautiful Poppies.

We offer the following varieties:

Beauty of Lirermore Deep crimson with black blotch ........................ $\$ 1.00$

Bracteatum Large scarlet, cup shaped bloom. Black blotch ..................... 1.00

Flanders Maroon shaded crimson ............. 1.00

Lula A. Neeley Bloom a deep ox-blood red on tall straight stems. The petals contin- 
ue to grow after opening which give it a longer blooming period. Very fine. Dr. Neeley's famous seedling ......... IIay Sadler Salmon pink with black markings Iasterpiece A large bloom of a peculiar shade of rose pink shading to salmon.......

Mrs. Perry Orange apricot. Lovely color, and very desirable ....................

Orientale Large crimson scarlet............ Prosperine Very large bloom of lively cerise scarlet..$\ldots \ldots \ldots \ldots \ldots \ldots \ldots \ldots \ldots \ldots$

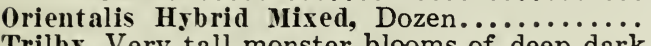

Trilby Very tall monster blooms of deep dark scarlet. Blooms after the others have

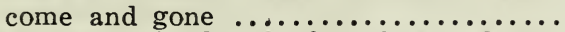

Wurtembergia Perhaps the finest Poppy grown. It is a beautiful ox-blood red and a very wonderful cushion of blue black, and black blotchs at base of petals. Unsurpassed in brilliance........ 3.00

We are very anxious to have as many gardens as possible have a number of these beautiful poppies, and as a special inducement will include a root of the entire collection with the exception of Orientalis Mixed for $\$ 16.00$.

We have also arranged a number of other collections which will add charm to your garden.

COLLECTION NO. 1

1 Wurtembergia ............. \$ 3.00

1 Bracteatum ................. 1.00

1 May Sadler ................ 1.00

1 Mrs. Perry ....................

1 Flanders ................. 1.00

$\$ 6.75$ For $\$ 5.00$

COLLECTION NO. 2

1 Wurtembergia .............. $\$ 3.00$

1 Masterpiece ............... 3.00

1 Orientale ..................... 1.00

1 Lula A. Neeley ............. 4.00

1 Mrs. Perry ................ .75

$\$ 11.75$ For $\$ 9.50$

COLLECTION NO. 3

1 Wurtembergia .............. $\$ 3.00$

1 Beauty of Livermore .......... 1.00

1 Prosperine ................. 2.00

1 Trilby .................... 3.00

1 Mrs. Perry $\ldots \ldots \ldots \ldots \ldots \ldots \ldots \ldots, \quad .75$

$\$ 9.75$ For $\$ 7.75$

COLLECTION NO. 4

1 Wurtembergia ..............\$ 3.00

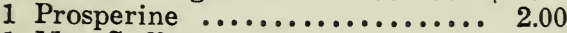

1 May Sadler ................ 1.00

1 Beauty of Livermore......... 1.00

1 Mrs. Perry ..................

1 Trilby $. . \ldots \ldots \ldots \ldots \ldots \ldots \ldots \ldots, 3.00$

1 Lula A. Neeley ............ 4.00 


\section{The Quest of Love}

By Sarah A. Pleas

O! garden mine, what pleasure waits Among your rows of gorgeous bloom For one who finds within your gates The title deeds to vast estates Of wealth, in beauty and perfume.

Today I come with new intent

To find the fairest flower of all

In form and color, size and scent,

$\mathrm{Ah}$, he will know how much is meant When by his name the flower I call.

How beautiful the flower must be To make it worthy of his name,

That other eyes than mine can see

What his dear memory is to me

Whose love I count as more than fame.

Here's one that's white with heart of gold A censer cup, for incense rare

And here is one of perfect mold

Whose silken petals seem to hold The secret of all beauty there.

Here's one as pure, as white as snow Fresh fallen on this world of ours

No hint of hue its petals show;

Shall I my name of names bestow On this to make it King of flowers?

Here's one so bright it glows like flame, What glorious color it has caught

From days and nights of June that came

To make it worthy the dear name

Which I have ever in my thought!

$\mathrm{Ah}$, look at this! So large, so sweet, A fluffy ball of pink and gold

In form and color all complete

Would we might stay the summer's feet To keep such beauty in our hold!

$\mathrm{O}$, what to choose! so fair are theySo rich in color, form, perfume!

But which the fairest, who shall say?

I needs must come another day

To find my garden's richest bloom.

So in a sweet bewilderment

I turn to leave my peony beds;

I have not found the flower I meant

To give the name I love, but spent

The morning hours where beauty spreads.

Such charm before me that I fear

I cannot choose among them all

Since each and all, I hold so dear

And yet-The one flower must be here.

That love by his dear name would call.

$\mathrm{O}$, here, I see! Close to me nods

A flower the color-soft blush rose,

So large, so sweet! Ah, by all odds,

This is a flower to please the Gods-

The best that in my garden grows!

My quest ends here, no farther I

Need seek among my peonies

To find the fairest flower, I cry

"O, royal bloom!" and he seems nigh

"I christen thee the Elwood Pleas." 